\title{
BMJ Open Does cognitive behaviour therapy affect peripheral inflammation of depression? A protocol for the systematic review and meta-analysis
}

\author{
Bing Cao (D) , ${ }^{1}$ Ruonan Li, ${ }^{1}$ Ling Ding, ${ }^{2}$ Jiatong Xu, ${ }^{1}$ Haijing Ma, ${ }^{1}$ Jie Liu, ${ }^{2}$ Jian Xue ${ }^{3}$
}

To cite: Cao B, Li R, Ding L, et al. Does cognitive behaviour therapy affect peripheral inflammation of depression? A protocol for the systematic review and meta-analysis. BMJ Open 2021;11:e048162. doi:10.1136/ bmjopen-2020-048162

- Prepublication history for this paper is available online. To view these files, please visit the journal online (http://dx.doi. org/10.1136/bmjopen-2020048162).

$\mathrm{BC}$ and $\mathrm{RL}$ contributed equally.

Received 24 December 2020 Accepted 08 November 2021

Check for updates

(c) Author(s) (or their employer(s)) 2021. Re-use permitted under CC BY-NC. No commercial re-use. See rights and permissions. Published by BMJ.

${ }^{1}$ Key Laboratory of Cognition and Personality, Faculty of Psychology, Ministry of Education, Southwest University, Chongqing, China

2Department of Pharmacy, JiangJin Central Hosptial of Chongqing, Chongqing, China

${ }^{3}$ Department of Health

Management, Zunyi Medical and Pharmaceutical College, Zunyi, Guizhou, China

Correspondence to

Dr Jian Xue;

xuejian912@163.com

\section{ABSTRACT}

Introduction Cognitive-behavioural therapy (CBT) is becoming the most commonly implemented and standard treatment for depression. Up to date, only a few numbers of studies have investigated the potential relationship between CBT and the change of inflammatory biomarkers in individuals of depression. And the results are inconsistent among studies. The current study aims to provide a comprehensive, systematic review of the association between CBT and changes of peripheral inflammation of individuals with depression, and clarify the alterations of inflammatory cytokines pre-CBT and postCBT treatment by meta-analysis, anti-inflammatory. Methods and analysis This study will be conducted in accordance with the Preferred Reporting Items for Systematic Reviews and Meta-Analyses guidelines. A systematic search of predetermined terms will be conducted with electronic databases of CENTRAL, MEDLINE, EMBASE and PsycINF0 from inception to July 2021. Database searches will be supplemented by expert contact, reference and citation checking, and grey literature. Primary outcomes of interest will be validated measures for levels of inflammatory cytokines pre-CBT and post- CBT treatment in individuals with depression. Hedges' $g$ will be used to represent the effect size.

Systematic review registration The protocol of current meta-analysis has been registered at the Open Science Framework (https://doi.org/10.17605/osf.io/tr9yh). Ethics and dissemination Formal ethical approval is not required by the National Ethical Review Board in China as primary data will not be collected. The results alterations of peripheral inflammatory cytokines pre-CBT and post-CBT treatment in individuals with depression will be disseminated through a peer-reviewed publication and inform the most up-to-date evidence of the roles of CBT treatment for depression.

\section{INTRODUCTION}

Depression is a severe and the most prevalent form of mental illness that characterised by neurocognitive deficits and disability. ${ }^{1}$ The disorder manifests in millions of individuals worldwide, and is a global health priority. The exploration of underlying mechanisms of depression are increasing over the past decades, none of them could clearly clarify
Strengths and limitations of this study

- This will be the first meta-analysis exploring the association between cognitive-behavioural therapy (CBT) and changes of peripheral inflammation of individuals with depression.

- The results could provide the most up-to-date evidence to assist in shared decision making between patients, caregivers and clinicians in treating the individuals with depression by using CBT.

- An insufficient number of original researches is a possible limitation.

- Potential high heterogeneity may cause selection bias and also decrease the reliability of our results.

the potential triggers of depression. ${ }^{2}$ In recent years, the inflammatory processes are considered to be important contributors to depression. A systematic review and metaanalysis confirmed that a high proportion of depressed individuals showed signs of inflammation. ${ }^{3}$ The potential bidirectional relationship of depression and inflammation were also clarified. ${ }^{4}$ For instance, early infection and autoimmune diseases are highly associated with high risk of depressive disorders in adulthood ${ }^{5}$; evidence from preclinical and clinical researches reached a consistency that the concentrations of peripheral proinflammatory cytokines significantly increase in individuals or animal models of depression. ${ }^{6}$ The inflammatory cytokines are mediators of environmental and genetic factors that may contribute to the development of depression from a biological perspective. ${ }^{7} \mathrm{~A}$ previous study suggested that inflammation may be involved in some certain medical conditions, and it may activate the pathogenesis of depression by interfering with the monoamine, glutamate and neurotrophic system. ${ }^{8}$ Dowlati et al's study reported that major depression leads to immune dysregulation and activation of the inflammatory 
response system. ${ }^{9}$ In addition, a growing body of evidence indicated that inflammation is thought to be an active process. The inflammatory process can affect multiple aspects of central nervous system function, and leads to behavioural changes in individuals with depression. ${ }^{10}$ The results of several meta-analyses have proved that depression is related to chronic low-grade inflammation. It is manifested by higher concentrations of peripheral $\mathrm{C}$ reactive protein (CRP), interleukin-6 (IL-6), and tumour necrosis factor- $\alpha$ (TNF- $\alpha$ ), compared with healthy controls. ${ }^{11}{ }^{12}$ Above findings have facilitated the development of the inflammatory hypothesis of depression, and have also predicted that inflammation plays a role in the formation, progression and perpetuation of depression. ${ }^{13}{ }^{14}$ Cognitive-behavioural therapy (CBT) is becoming the most commonly implemented and standard treatment for depression..$^{15}$ Briefly, CBT is based on the premise that false beliefs and negative thoughts are the main causes of depression. The individuals develop strategies for managing and preventing depressive symptoms by monitoring mood symptoms and using a repertoire of coping skills to manage stress in $\mathrm{CBT} .{ }^{16}$ A meta-analysis found that there is no difference in treatment effects of CBT and second-generation antidepressants, either alone or in combination. ${ }^{17}$ Recent published literatures illustrated that similar with antidepressant treatment, CBT may also contribute to reduction of chronic low-grade peripheral inflammation. ${ }^{18}$ However, the underlying biological processes of the CBT effects on depression are still very limited.

To date, only a few numbers of studies have investigated the potentially anti-inflammatory effects of CBT for depression. Results are inconsistent among studies. For example, some researches pointed out that peripheral IL-6 was decreased after CBT, while no significant changes were founded in other studies. ${ }^{19}$ Kéri et al reported that in adults with a first episode of depression, 16 weeks of CBT alone was associated with a reduction in TLR-4 signalling, but no change in TLR-2 signalling, IL-6, or CRP levels. This study suggested that it took longer time or other mechanisms for them to normalise. ${ }^{20}$ Additionally, whether the alterations of inflammatory cytokines associated with improvements in depression after CBT treatment is also not well established. So far it is worthy to conduct a systematic review and meta-analysis to summarise the most updated research results for the role of CBT treatment in inflammation of depression.

\section{AIMS AND OBJECTIVES}

The current study aims to provide a comprehensive, systematic review of the association between CBT and inflammation of individuals with depression, and clarify the alterations of inflammatory cytokines pre-CBT and post-CBT treatment by meta-analysis. From the previous evidence, we hypothesised that some inflammatory cytokines, such as CRP, IL- 6 , TNF- $\alpha$, may decrease after the CBT intervention.

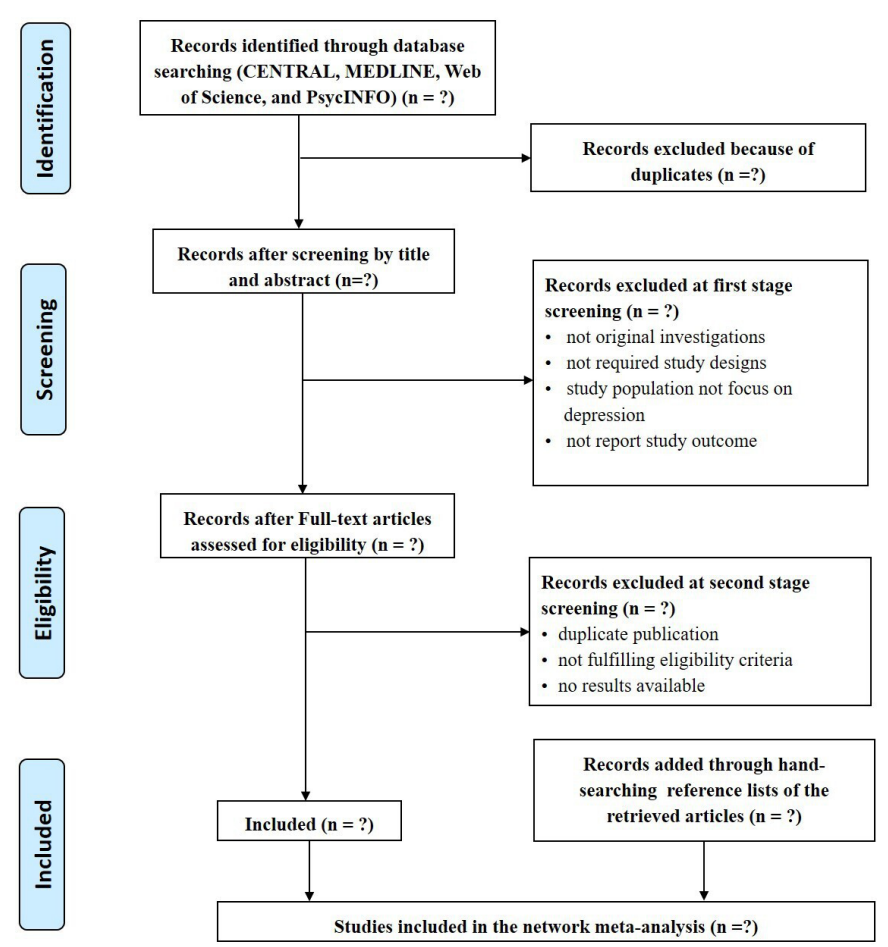

Figure 1 PRISMA flow diagram of study selection process. PRISMA, Preferred Reporting Items for Systematic Reviews and Meta-Analyses.

\section{METHODS}

\section{Search strategy}

The systematic review and meta-analysis will be conducted and reported in accordance with the Preferred Reporting Items for Systematic Reviews and Meta-Analyses (PRISMA) statement. ${ }^{21}$ Figure 1 summarises the study selection as a PRISMA flowchart. A systematic search of predetermined terms will be conducted with electronic databases of CENTRAL, MEDLINE, EMBASE and PsycINFO from inception to July 2021. The keywords of our search strategy will be the key terms mapped to subject headings for (1) depression (major depressive disorder, depression, mood disorder, dysthymic disorder); (2) CBT (psychotherapy, cognitive therapy, cognitive behavioural therapy); (3) inflammatory cytokines (cytokine, IL, chemokine, interferons, tumour necrosis factor, as well as the specific inflammatory biomarkers). The search strategy of PubMed is shown in table 1.

\section{Selection criteria}

The studies conducting the within-group comparisons of the peripheral levels of cytokines and chemokines in participants with depression at baseline and after CBT will be included in the current meta-analysis. Study eligibility for the inclusion criteria will be assessed by the approach of Population, Intervention, Comparison, Outcome and Study Design. Moreover, according to the quality assessment recommendation of Cochrane Collaboration, we will use Newcastle-Ottawa Scale to evaluate the quality of the included literatures. ${ }^{22}$ Population: adult subjects $(\geq 18$ years old) meeting the major depression diagnoses by the 


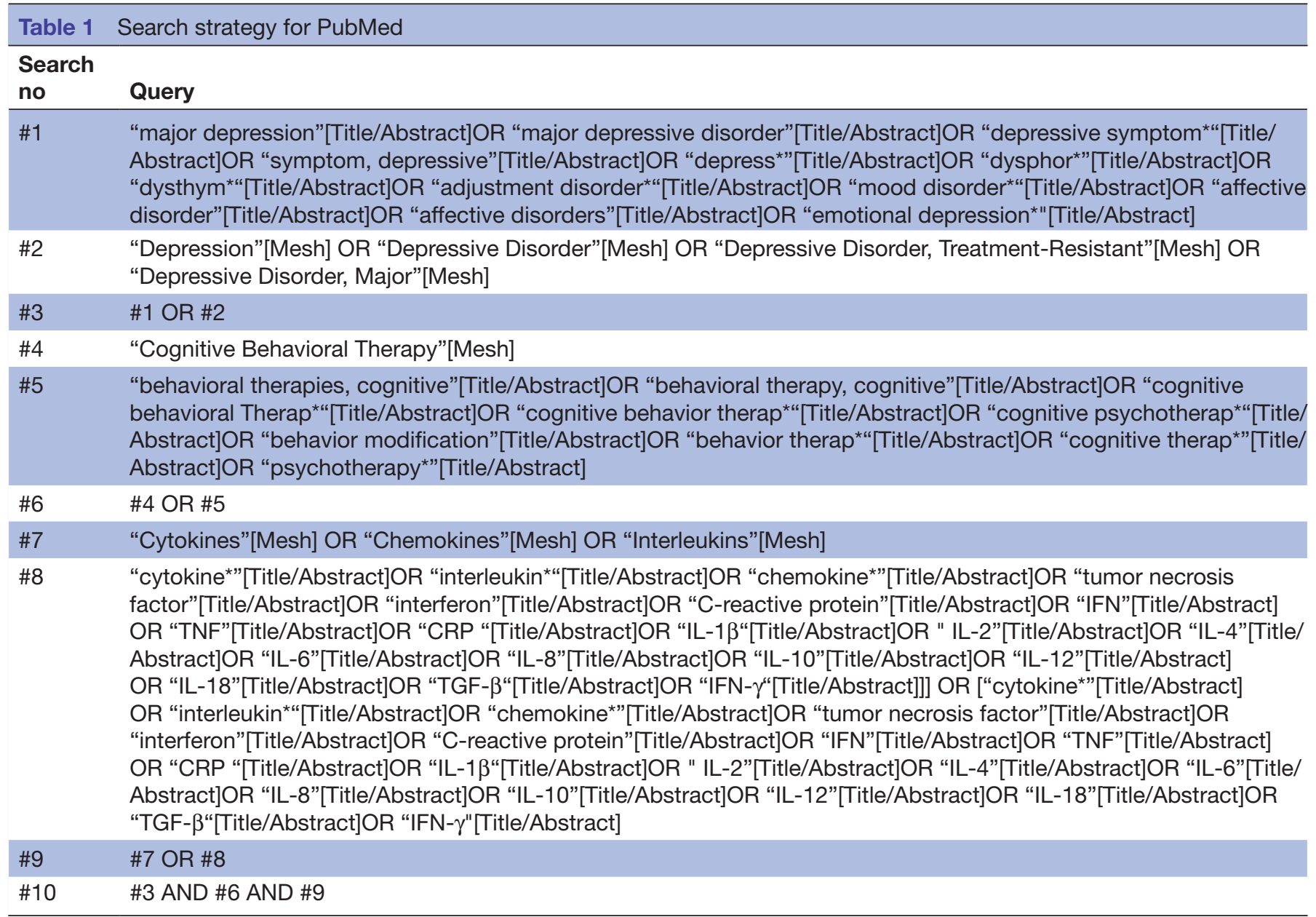

Diagnostic and Statistical Manual of Mental Disorders (no restrictions on editions) or International Classification of Diseases and Related Health Problems criteria; Intervention and Comparison: assessed the results of peripheral inflammatory cytokines (eg, IL-6, CRP or IL-10) before and after CBT. Outcome: reported mean or median resting levels of inflammatory cytokines in saliva, blood or urine was reported before and at least once after starting CBT or the effect size (ES) could be calculated from the reported results. Study Design: randomised controlled trial, open-label study or longitudinal study with pretestpost-test design. Exclusion criteria of the studies will meet if they (1) did not focus on evaluating inflammatory cytokines levels on depression; (2) only reported stimulated levels of cytokines; (3) were repetitive publications from the same datasets by the same or different authors and (4) included participants who have been receiving pharmacological treatment in the past 1 month.

\section{Outcome measures and data extraction}

The outcome for this meta-analysis will be the changes of inflammatory cytokines pre-CBT and post-CBT treatment in individuals with depression, which were measured by the standard mean differences (SMDs) their concentrations. Paired investigators (JX and $\mathrm{HM}$ ) will independently select the studies, reviewed the main reports and supplementary materials, extracted the relevant information. All reference lists of the retrieved articles will be reviewed to identify the potential studies. The following information will be extracted from each study: first author, publication year, study design, country, geographical location, age, sex, intervention, duration of intervention, whether it is a major depressive episode, inflammatory biomarkers measured, type of sample specimen required for test, sample detection method, sample size, the mean levels of subjects' peripheral inflammatory cytokines and SDs before and after CBT treatment.

\section{Statistical analysis}

Only the inflammatory cytokines with sufficient numbers of studies $(\geq 3)$ will be performed the meta-analysis. Analyses will be performed for pretest and post-treatment of CBT. The main analysis will be conducted with a random effects model. Forest plots will be used to estimate the alteration of the levels of inflammatory cytokines pretreatment and post-treatment CBT, which will be evaluated by SMD with a $95 \%$ CI. The ES is represented as Hedges' g in order to adjusted for a potential bias wo overestimate the ES in small samples. According to the statistical power analysis for the behavioural sciences (second edition), the ES is judged using the values of $0.2,0.5$ and 0.8 for small, medium and large (Cohen, 1988). In addition, we 
will conduct Bonferroni adjustment for multiple testing in meta-analysis, which means we will produce a rejection p value of 0.05 divided by the total number of outcomes. ${ }^{23}$ The heterogeneity across the studies will be evaluated by $\chi^{2}$ statistics and $\mathrm{I}^{2}$ test. A value of $\mathrm{p}<0.10$ or $\mathrm{I}^{2}>50 \%$ indicated that the heterogeneity of effect estimates within each group of studies was statistically significant,${ }^{24}$ and it also shows that the percentage of the variability in effect estimates owes to heterogeneity rather than chance. Furtherly, the subgroup analysis and meta-regression will also be performed to investigate the source of the heterogeneity, and the potential influence of included characteristics of the studies on the pooled ES. Sensitivity analysis was performed to strengthen the results and investigate whether any single study would have an effect on the heterogeneity of total measurements in each meta-analysis. Additionally, the positive and negative results may not be equally likely to get published, thus the funnel plot with Begg's test and Egger's test will be used for testing the publication bias. If publication bias will be found, then the trim and fill method would be used to both identify and correct the asymmetry of funnel plot. All two-tailed $\mathrm{p}<0.05$ will be defined as statistical significance. All the data analyses will be conducted using Stata (V.15.0, StataCorp).

\section{Patient and public involvement}

Patients were not involved in the development of this systematic review protocol. The data for this systematic review will be collected from previously published studies.

\section{Ethics and dissemination}

Formal ethical approval is not required, as primary data will not be collected with the systematic review and metaanalysis. Data from previously published studies will be retrieved and analysed. This study including protocol development will run from October 2020 to October 2021. The results will be disseminated through a peerreviewed publication and inform the most up-to-date evidence of the roles of CBT treatment for depression.

\section{DISCUSSION}

\section{Presentation of results and reporting}

Our current systematic review and meta-analysis will provide comprehensive evidence for the association between CBT and inflammation of individuals with depression. We will use the PRISMA guidelines and checklist in the publication process. The quantitative data will be summarised and presented in tables, forest plots and charts. The alterations of inflammatory cytokines preCBT and post-CBT treatment in individuals with depression will be presented.

\section{Potential resources of limitations}

The current study is anticipated to have some limitations. First, we might not find a sufficient number of original researches to perform the analyses. Second, the potential high heterogeneity between studies in the exposure of interest and restriction to studies in English language will lead to selection bias and also decrease the reliability of our results.

To the best of our knowledge, this will be the first meta-analysis exploring the association between CBT and peripheral inflammation of individuals with depression. From our findings herein, we can provide the most up-todate evidence to assist in shared decision making between patients, caregivers, and clinicians in treating the individuals with depression by using CBT and provide a foundation for future studies in this area.

Contributors $\mathrm{BC}, \mathrm{JX}$ and $\mathrm{HM}$ conceived the study and developed the search strategy. BC drafted the protocol and tested the search strategies in consultation with a librarian. RL, LD and JL provided advice on the protocol. All authors critically revised the protocol for methodological and intellectual content and have read and approved the final manuscript.

Funding This research work is supported by funding from Southwest University (SWU019039), The Science and Technology Support Program of Guizhou Science and Technology Department (grant numbers (2020) 4Y183); The Science and Technology Support Program of Zunyi Science and Technology Bureau (grant numbers (2020)22).

Disclaimer The funding bodies had no part in either the study design, conduct, analysis or interpretation of this study.

Competing interests None declared.

Patient and public involvement Patients and/or the public were not involved in the design, or conduct, or reporting, or dissemination plans of this research.

Patient consent for publication Not applicable.

Provenance and peer review Not commissioned; externally peer reviewed.

Open access This is an open access article distributed in accordance with the Creative Commons Attribution Non Commercial (CC BY-NC 4.0) license, which permits others to distribute, remix, adapt, build upon this work non-commercially, and license their derivative works on different terms, provided the original work is properly cited, appropriate credit is given, any changes made indicated, and the use is non-commercial. See: http://creativecommons.org/licenses/by-nc/4.0/.

ORCID iD

Bing Cao http://orcid.org/0000-0001-5963-2676

\section{REFERENCES}

1 Ang Y-S, Frontero N, Belleau E, et al. Disentangling vulnerability, state and trait features of neurocognitive impairments in depression. Brain 2020;143:3865-77.

2 Mclntosh AM, Hall LS, Zeng Y, et al. Genetic and environmental risk for chronic pain and the contribution of risk variants for major depressive disorder: a family-based mixed-model analysis. PLoS Med 2016;13:e1002090.

3 Osimo EF, Baxter LJ, Lewis G, et al. Prevalence of low-grade inflammation in depression: a systematic review and meta-analysis of CRP levels. Psychol Med 2019;49:1958-70.

4 Beurel E, Toups M, Nemeroff CB. The bidirectional relationship of depression and inflammation: double trouble. Neuron 2020;107:234-56.

5 Benros ME, Waltoft BL, Nordentoft M, et al. Autoimmune diseases and severe infections as risk factors for mood disorders. JAMA Psychiatry 2013;70:812-20.

6 Kim Y-K, Na K-S, Myint A-M, et al. The role of pro-inflammatory cytokines in neuroinflammation, neurogenesis and the neuroendocrine system in major depression. Progress in NeuroPsychopharmacology and Biological Psychiatry 2016;64:277-84.

7 Song C, Wang H. Cytokines mediated inflammation and decreased neurogenesis in animal models of depression. Progress in NeuroPsychopharmacology and Biological Psychiatry 2011;35:760-8.

8 McNally L, Bhagwagar Z, Hannestad J. Inflammation, glutamate, and glia in depression: a literature review. CNS Spectr 2008;13:501-10. 
9 Dowlati Y, Herrmann N, Swardfager W, et al. A meta-analysis of cytokines in major depression. Biol Psychiatry 2010;67:446-57.

10 Irwin MR, Miller AH. Depressive disorders and immunity: 20 years of progress and discovery. Brain Behav Immun 2007:21:374-83.

11 Köhler CA, Freitas TH, Stubbs B, et al. Peripheral alterations in cytokine and chemokine levels after antidepressant drug treatment for major depressive disorder: systematic review and meta-analysis. Mol Neurobiol 2018;55:4195-206.

12 Więdłocha M, Marcinowicz P, Krupa R, et al. Effect of antidepressant treatment on peripheral inflammation markers - a meta-analysis. Progress in Neuro-Psychopharmacology and Biological Psychiatry 2018;80:217-26.

13 Köhler-Forsberg O, N. Lydholm C, Hjorthøj C, et al. Efficacy of antiinflammatory treatment on major depressive disorder or depressive symptoms: meta-analysis of clinical trials. Acta Psychiatr Scand 2019;139:404-19.

14 Husain MI, Strawbridge R, Stokes PRA, et al. Anti-inflammatory treatments for mood disorders: systematic review and meta-analysis. Journal of Psychopharmacology 2017;31:1137-48.

15 Lopez MA, Basco MA. Effectiveness of cognitive behavioral therapy in public mental health: comparison to treatment as usual for treatment-resistant depression. Adm Policy Ment Health 2015;42:87-98

16 Pearlstein JG, Staudenmaier PJ, West AE, et al. Immune response to stress induction as a predictor of cognitive-behavioral therapy outcomes in adolescent mood disorders: a pilot study. J Psychiatr Res 2020;120:56-63.
17 Amick HR, Gartlehner G, Gaynes BN, et al. Comparative benefits and harms of second generation antidepressants and cognitive behavioral therapies in initial treatment of major depressive disorder: systematic review and meta-analysis. $B M\lrcorner$ 2015;351:h6019.

18 Lopresti AL. Cognitive behaviour therapy and inflammation: a systematic review of its relationship and the potential implications for the treatment of depression. Aust N Z J Psychiatry 2017;51:565-82.

19 Felger JC, Lotrich FE. Inflammatory cytokines in depression: neurobiological mechanisms and therapeutic implications. Neuroscience 2013;246:199-229.

20 Kéri S, Szabó C, Kelemen O. Expression of toll-like receptors in peripheral blood mononuclear cells and response to cognitivebehavioral therapy in major depressive disorder. Brain Behav Immun 2014;40:235-43.

21 Moher D, Liberati A, Tetzlaff J, et al. Preferred reporting items for systematic reviews and meta-analyses: the PRISMA statement. PLoS Med 2009;6:e1000097.

22 Lo CK-L, Mertz D, Loeb M. Newcastle-Ottawa scale: comparing reviewers' to authors' assessments. BMC Med Res Methodol 2014;14:45

$23 \mathrm{Ng} \mathrm{A}$, Tam WW, Zhang MW, et al. IL-1 $\beta$, IL-6, TNF- $\alpha$ and CRP in elderly patients with depression or alzheimer's disease: systematic review and meta-analysis. Sci Rep 2018;8:12050.

24 Cao B, Wang D-F, Xu M-Y, et al. Lower folate levels in schizophrenia: a meta-analysis. Psychiatry Res 2016;245:1-7. 